\title{
Technical issues for IP-based telephony in Nigeria
}

\author{
A. A. Ojugo. ${ }^{1}$, R. Abere. ${ }^{1}$, B. C Orhionkpaiyo. ${ }^{1}$, E. R. Yoro ${ }^{2}$, A. O. Eboka ${ }^{3}$ \\ ${ }^{1}$ Department of Mathematics/Computer, Federal University of Petroleum Resources Effurun, Delta State \\ ${ }^{2}$ Department of Computer, Delta State Polytechnic Ogwashi-Uku, Delta State \\ ${ }^{3}$ Dept. Of Computer Sci., Federal College of Edu, (Technical), Asaba, Delta State
}

\section{Email address:}

ojugo_arnold@yahoo.com(A.A. Ojugo), arnoldojugo@yahoo.com(A. A. Ojugo), areuben2001@yahoo.com(R. Abere), orhionkpaiyo@yahoo.com(B.C Orhionkpaiyo),rumerisky@yahoo.com(E. R. Yoro), andre_y2k@yahoo.com(A. O. Eboka)

\section{To cite this article:}

A. A. Ojugo., R. Abere., B. C Orhionkpaiyo., E. R. Yoro, A. O. Eboka. Technical Issues for IP-Based Telephony in Nigeria. International Journal of Wireless Communications and Mobile Computing. Vol. 1, No. 2, 2013, pp. 58-67. doi: 10.11648/j.wcmc.20130102.11

\begin{abstract}
Data transmission in IP-networks, with all its challenges and associated prospects, is today a major milestone in the convergence of information and communication technology (ICT). IP-telephony simply refers to the data transfer that encapsulates data (in forms of audio, data, voice and video) media or traffic so that they are transported over data network via IP network technologies. IP telecommunications defines a wider range of applications, technologies and convergence networking that refers to a multi-service network that allows the integration of data, voice and video solutions onto a converged network infrastructure via the use of hardware, software and open-source apps/protocols (such as H.323, Megaco/H.248 signaling and transport protocols) used to setup, control and manage both data, voice and video sessions. The study selected area is the Federal University of Petroleum Resources Effurun in Delta State, in which the VoIP was implemented and issues in its implementation were discussed. These include: delay, packet loss and jitters. Jitters and Packet loss can be curbed via increase in bandwidth allocation; latency can be minimized but not totally eradicated by constantly upgrading the network equipment in terms of speed. The many benefits of IP communications cannot be over-emphasized as it includes advanced dial tone, rich media streaming, unified messaging, IP contact center etc while providing the advantages such as resilience, economy, flexibility, mobility and productivity for users. Even with its many pending challenges, IP communications continues to proffer advancements in mobile computing, apps to support mobile users and telecommuting apps that provide users the much needed merits with soft-phones, soft agents and VPN services. It is recommended that an organization intending to harness its full potentials should join forums and user-groups that will constantly update them such as Multi-Switch Forum (MSF) - in a bid to help them improve the efficiency and effectiveness of their IP implementation.
\end{abstract}

Keywords: Communications, Data, Media, Traffic, Voice, Data Services

\section{Introduction}

IP-based telephony is simply data transfer or transport of data (in forms of voice, file, audio and video) over telecommunication media using the Internet Protocol (IP). Communication is the key to fast development with shared ideas, giving birth to new ones to provide a platform for exponential innovation in ICT. The development in IP has drastically provided a new, different alternative set for provision of long distance calls and data transfer with 3-basic factors: (a) IP is a packet network that only uses resources on need, (b) methods exist that allows transmission of voice in a highly compressed format while retaining quality that the consumer demands cum allows the integration of speech, and (c) technology is available that allows integration of telephone networks with the speech compression systems and in turn, with IP. Thus, bringing closer the intent and concept of IP universality namely low cost, speech compression, quality and ease of implementation for IP long distance calls [10, 14, 16].

The birth of 3G-telecommunications has brought about use of interactive services, originally made possible via PSTN (public switched telephone network) - so that transfer is now expansive (over long distances) without video interactions. The advent of IP reinvents communications via new technologies, allowing users to exchange data in real time - via IP telephony and alternatives, which are fast gaining wide acceptance as open-source solutions to users as well as opening new functionalities for markets that previously required expensive PBXs - noting that IP telephony is more, software-based rather than hardware implemented [1, 4]. 


\subsection{PSTN Overview}

PSTN are dumb devices driven by an intelligent network - with signals sent between phone switches to indicate terminal status involved in calls and to connect or terminate calls.

PSTN/Traditional PBX still dominate the market, helping to provide allied and various ICT solutions to small/medium enterprises. PSTN employs devices such as plain old telephones (POTs), phone lines, fibre-optics, cables, microwave and satellites, cellular and undersea telephone cables, all aimed at interconnecting switch-centers, allowing all the users across the world to interact [8].

Its signals are carried over separate data network known as Common Channel Signalling (CCS) that uses Signalling System 7 protocol and Signalling Control Points (SCP) as its database to provide necessary data needed for advanced call processing. It also has a Centrex (Service Provider's end) that provides business class telephony feats, as built on a carrier's central office switch, allowing users to have either an analog or digital phone [3, 10]. Such telephones are connected via an copper pair back to a central office switch via:

1. Centrex lines (copper pairs access nodes linked to a central switch with PSTN Connectivity

2. Analog and/or digital handsets or terminals

3. Centrex feats such as caller ID, call centre feats, etc and an optional voice mail

\subsection{Converged Network}

The basic foundations of a converged network is in its capabilities and tools that allows users flexibility, secure and cost effectively data transfer services (in form of or a combination of data, voice and video) packets across the same transmission links via the same switching, routing and gateway platforms. Thus, the converged-network is more fault tolerable, flexible, secure, resilient, scalable and showcases easy manageability of data services and network devices [4, 16] - as its applications use open source underlying intelligent network services to ensure data transfer service quality, availability, reliability and security [5]. With electronic data systems invading every frontier, access becomes increasingly bothersome to have users being tethered with wires. Thus, the need for wireless devices with merits such as: (a) low-cost deployment, (b) broadcast the same data to many locations simultaneously, (c) deployment ease in hostile environment, and (d) mobile communication. Its demerits: (a) lesser data rates, (b) lesser reusable frequencies, and (c) more susceptible to interference [5].

PSTN today, is being replaced with IP, packet networks (with PSTN still used by non-IP devices connected to such network). Thus, PSTN conveniently support various data services via SCP and by Internet devices wishing to connect to such PSTN network. Conversely, IP telephony is a call process that uses signal technology built on Open IP-standards, providing users with end-to-end communication or data transfer (file, data, voice and video formats) - to aid data transfer services for public carrier networks and Internet users in general with an inter-operable networks [7].

[2] Note IP telephony involves a large family of communication standard to deliver voice and video services via open packet network and uses H.323 protocol to setup, control and manage sessions. The many benefits of IP-network as easily deployed services over legacy PSTN includes:

- IP Telephony allows call processing services to be located anywhere on network and use packet networks rather than TDM for services.

- $\quad$ Allows service delivery over converged network so that dual cabling and network equipments for connections to PBX or IP-PBX are not required.

- Carry traffic across different areas and various vendors spanning various countries - interfacing a variety of Internet and Telephone technolgies more flexibly, with greater benefits at reduced cost of implementation and operation.

Thus, the converged network simply helps to extend the capabilities of such an intelligent IP network over or into a PSTN network (when built to use underlying network) protocols and are based upon a server or network appliance $[9,15]$.

\subsection{Applications of IP Telephony}

[21] Note IP-telephony as applied in various forms:

1. Private users who employ IP telephony in VoIP for end-to-end voice calls over the Internet as they constantly trade quality, features and reliability for low cost. The density of users in this group is low even though many users (globally) take advantage of the technology, when compare with the PSTN call volumes.

2. Business users on private networks provided by telecomm/datacom providers - with high quality, feature rich services and reliability that comes at high cost. In contrast, when compared to users of PSTN call volumes - they are small.

3. IP trunking solution are used by long haul voice providers to connect islands of PSTN together to private IP networks, and users access these services via traditional black phones but the data is carried over an IP-network.

These applications continues to have its place in the future of IP networks and in general, telecommunications; But, we are yet to deal with issues of how the wider PSTN can be mitigated to an end-to-end voice infrastructure. Simply put, how do we provide a voice over IP solution that scales to PSTN call volumes and offer PSTN call quality, equivalent services and support such new innovative services.

\subsection{Benefits of IP-Network}

The benefits of converged intelligent network are derived 
from its fundamental capabilities to provide these advantages:

1. Economy - Traditional PSTN uses expensive legacy systems as connecting elements, apps and technologies like DS1/DS0 line cards, trunk cards and digital signalling technologies. Conversely, IP networks allow users to build data services via Ethernet economics and Moore's law for rapid advances in computing performance. A merit of IP-network over traditional PSTN is cost in connecting of enterprise PBX system to PSTN via ports, cards and circuits. Thus, cost is lesser to provide connections to other sites and to other apps [17].

2. Flexibility - PSTN element/app(s) are mostly proprietary, monolithic and restrictive. Whereas IP network connections are made from virtual reach with resources distributed on demand to anywhere needed, and economies are gained via centralized gateway and server resources. Use of many types of media and applications to be brought together to facilitate communication. It supports broadband voice, front/back office integration and apps, outsourcing operation, mobility requirements, centralised management, telecommuting, moves/adds/changes, extension mobility, desktop integration and automation, enterprise directories and takes advantage of a plethora of emerging web services such as instant messaging, presence and mobility [19].

3. Security - It aims at achieving a higher degree of security for secure data transfer that are vastly superior over legacy voice technologies; while deploying and integrating wireless LAN apps, video surveillance, IP video on demand, streaming and rich media conferencing applications $[17,18]$.

4. Resilience - With business continuity and disaster recovery high, resiliency of connectivity and abilities provided by IP network keeps an organization connected make it an ideal candidate for survivable services. Its redundancy is built into intelligent layer2 and layer3 networking technologies and apps. Clustering and hot standby technologies, fault tolerant storage technologies like RAID, dual power supply and UPS systems are now common in the industry. IP offer superior failover, self-healing and redundant abilities that are easy to deploy, open standards based, and can support an organization's communication services. It offers reliability, availability and superior alternatives over legacy PSTN that are far more expensive, and are unable to provide same overall system resiliency needed for as broad a range of services and apps as in IP-network [18-19].

5. Productivity - Its focus shift from cost savings to enabling users become more productive with apps to help accomplish higher quality communications more quickly and easily, has yielded a network of phone apps that employ any existing web or enterprise database on an IP network. Thus, end-users can take advantage of emerging web innovations, enterprise directories, e-mails, voice mail, fax, and general tools for programming communication rules. Voice recognition and soft-phone support via user desktops can be added to an IP-communications environment. While some of these possibilities exist in legacy PSTN, they are more expensive, less scalable, and more difficult to deploy [6].

\section{Objectives}

The study aims to address IP telephony issues implemented at Federal University of Petroleum Resources Effurun, Delta State, Nigeria; while ensuring that its many benefits are fully harnessed.

\section{Methods and Materials}

A critical, structured analysis of the existing hybrid PSTN/PBX system indicates that these must be addressed before effective implementation namely:

1. Difficulty to accomodate differences in disparate technologies and equipment

2. Diffuculty to traverse geographic boundaries

3. Manage many sites centrally

4. Change the way resources are used on a network

5. Traverse regulatory boundaries

6. Deliver such new communication services using different media types

7. Provide level of integration, ease of use, access and management found in IP telephony systems.

\subsection{Network Planning}

Planning an IP-based network on FUPRE's existing hybrid PSTN/PBX - took into account that services must be accessed by 5000 users (both staff and students). The Nigerian University Commission (NUC) note that to aid effective learning, each department is encouraged to have a teacher-student ratio of $1: 25$; FUPRE has a ratio of $1: 45-$ except in General Studies and its related courses where number exceed. Currently, with eleven departments, four levels in Science and five levels in Technology respectively - the institution has a total of 2300 students, and 1210 staff (academic and non-academic) - a total of 5000-subscribers simultaneously.

\subsection{Network Components}

The Federal University of Petroleum Resources has 2-campuses of $3 \mathrm{~km}$ apart. The VoIP design implemented will cater for 5000-user simultaneously and effectively provide the needed IP solution. This can be deployed in 
many different segments but mostly on backbones and enterprise networks. We adopt [3] next generation VOIP end-to-end architecture with additional constraints, implemented on FUPRE as in fig 1. There are three (3) service providers (SP) with varied functions as: (a) Service Provider 1 - will offer local access as a LEC to support IP phones and IP PBX systems using SIP and POTS phones via either an access gateway (Next-Gen DLC) or a subscriber gateway using the H-248 or MGCP protocol, (b) Service Provide 2 - will act as an interchange to support SIP and
SIP - T or BICC signaling through its network, and (c) Service Provider 3 - will offer local access as LEC that only supports POTS phones using an Access Gateway. SIP signaling is supported but is terminated by the SIP server rather than using a SIP phone or other CPE devices. The functional specification of the network components, depends on the particular network architecture and LAN cabling - as some of these components may be combined into a single solution.

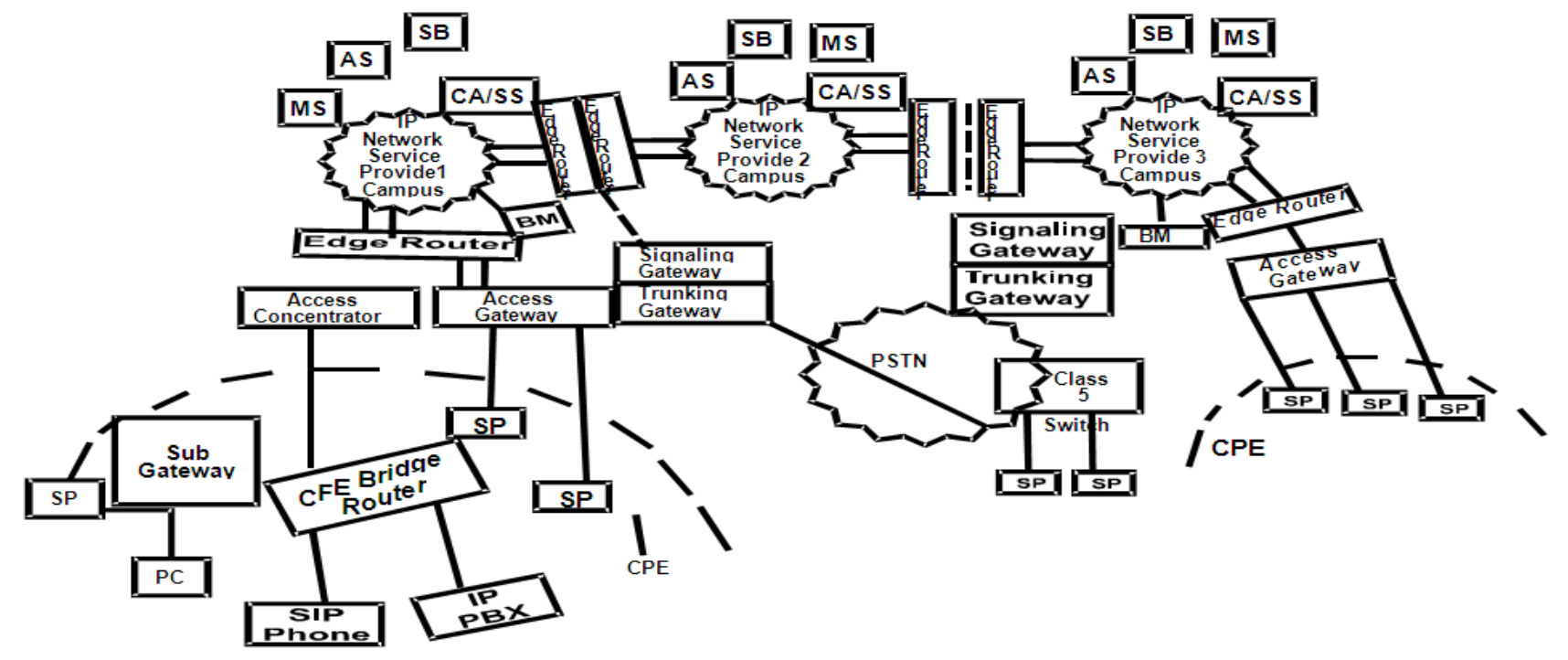

Fig 1. IP-Network Solution in FUPRE

For example, a combined signaling and trucking gateway. The vision of the project still remains - implementation of IP telephony, while the scope remains FUPRE and the function of each network component is described as thus:

1. Call Agent and SIP: Call agents (may include service logic to) provide an IP network with call logic, call control functions, maintains state of every call on network and offer supplementary services (like Call ID, waiting, forwarding etc) and also interact with servers to supply services that are not directly hosted on call agents. They participate in signaling, device control flows and provide details of each call to support building and reconciliation. Call agents are also called soft-switches, media gateway controller and call controllers - all of which conveys the emphasis on its ability to maintains call state as the common function.

2. SIP server provides equivalent function as the call agent with its primary role being to route and forward SIP requests, enforce policies (like care admission control) and maintain call details records; while SIP client conversely, provides similar function but originates and terminates SIP signaling rather than forwarding it to a SIP phone or other CPE devices.

3. Service Broker (SB) provides service distribution; coordination and control between all the application servers, media server, call agents and services that exist on alternate technologies (that is, create a parlay gateway and SCPS). It also allows consistent repeatable approach for controlling applications in conjunction with their service data and media resources to enable services and allow service to be reused with other services, to create value added services.

4. Application Server (AS) provides the service logic and execution for one or more applications or services that are not directly hosted on the call agent. It may provide voice mail or conference calling facilities typically, the call agent will route to the appropriate application server when a service is involved that which call agent itself cannot support.

5. Media Server use control protocol such as H.248/Megaco/MGGP, as supervised by a CA or AS to provide functions such as: (a) Play announcement, (b) provide 3-way call support, (c) Codec transcoding and voice detection, (d) Tone detect/generation, (e) Interactive Voice Response processing and (f) jaw processing.

6. Signaling/Trunking Gateway: Signaling gateway acts as a gateway between the call agent signaling and SS7 protocol-based PSTN, to provide the required signaling translation and a cross platform for and between different packets carrier domains. 
7. Trunking gateway provides the gateway between the carrier IP network and Time-Division Multiplexing PSTN. Thus, provides transcoding from the packet based VOIP onto a TDM network (a reason for our choice of design - merging both into one single solution). Trunking gateway is controlled by a Call Agent via a device control protocol (H.248/MGCP).

8. Access Gateway/Concentrator: Access gateway provides support for POTS; while access concentrator helps to terminate service provided from WAN links used. Thus, in a DSL (Digital Subscriber Link) network, its function is to combine the capabilities of DSLAM (Digital Subscriber Line for Autonomous Machines) with direct POTS (plain old telephone systems).

9. Edge Router helps to route IP traffic onto the carrier backbone network and provides many other functions when combined with Access Concentrator into one single solution.

10. Bandwidth Manager is responsible for providing the required QOS (Quality Of Service) from the network, setting up and teasing down of bandwidth within the network as well as controlling the access of individual calls to this bandwidth while also helping to install the appropriate policy in edge routers to police media/data flow on a per call basis.

11. Gateways/Router/Bridge helps terminate user sessions from the WAN link (such as DSL, T1, fixed wireless, cable) and provides both voice ports and data connectivity. Bridges/routers and gateways perform same function with a simple difference that bridges/routers does not provide any native voice support, though voice services such as SIP phones can be bridged/routed via the device(s).

12. IP Phone/ PBX: IP phones, softphones and PBX systems provide voice services. They interact with the Call Agent/SIP Server in signaling protocol SIP, H.323 or a device control protocol Megaco (i.e. H.248).

In summary, these components are required to build the converged, and can be grouped into four as:

a. Intelligent Network infrastructure (like switches, routers, gateways etc), services, software and protocols - mostly dominated at SP's end (in hosted IP solution) to form the physical infrastructure and deliver intelligent network services such as security, QOS and resiliency.

b. Application provides new capabilities via integrated (audio-data-voice video) apps with improved call feats such as Media conferencing. Unified messaging and IP contacts. All of which work better in a secure IP network due to the trunkless/portless nature of IP telephony to use such new innovations and apps that continues to yield greater productivity.

c. End Point Client Devices are the access point from where users take advantage of IP network apps such as IP-phones, soft-phones (desktop systems with speakers), PDA (Personal Digital Assistants), mobile phones etc.

d. Call Processing - is the software that drives it all so as to run effectively network appliance servers on third party servers.

\section{Implementation Issues}

IP network growth has brought about radical transformation and improvement in the market, technology and open source software, allowing them to gain wide acceptance. The incremental design of implementation adopted, the technical issues experienced that threatens service quality are $[4,5,14]$ :

\subsection{Service Set}

A crucial decision facing IP network deployment is the service set and design to be supported, which can be either minimal set, full scale PSTN equivalence or advance services for operators and carriers wishing to replace their current infrastructure with a new converged network for all subscribers. An important feat in the design is the choice of user terminal to be supported by the services to be offered to include POTS/black phones, IP phones, PBX and key systems, PC soft-phones (with web apps) etc.

\subsection{Security}

PSTN became resistant to security attacks with the advent of SS7 out-of-band signaling. IP nets are more susceptible to attacks to address 3-issues:

1. Invasion of Privacy - Callers in PSTN expect calls are private with no third party eavesdrop. PSTN achieves this via a physical security mechanism (wire from a user's home is only connected to local exchange or digital loop carrier and cannot easily accessed). Whereas, IP network uses different encryption measure to cater for such security issues via its cable/wireless media. E.g A5 cipher used in GSM or CDMA.

2. Denial of Services attack prevents a legitimate user access to the network feats and services. Though rare and extremely difficult in PSTN; But, are common in IP networks. Example includes sending false signaling message so that a call agent is fooled and bombarded with pings from a soft phone or other packets so frequently that it has no spare processing power to process legitimate request. A consequence of this is that sometimes - the soft-phone(s) can no longer pull data from the IP network. Also, hacking a subscriber gateway to send ftp or other data traffic as high priority voice traffic.

3. Theft of Service - is aimed at SP where the attacker simply wants to use a service without paying for it. Its most common form in PSTN is called subscriber fraud - where a user sets up an account with a 
service provider (SP) using false billing data such as stolen credit card. other forms are more technical that utilizes black boxes or similar to fool the network into providing free service. With VoIP, bandwidth is still a limited resource even with low packet loss and jitters required for good voice quality.
Thus, converged network needs to be protected from users who misuse high-priority bandwidth. E.g. Two SIP user agents can setup a direct call between them, to access high-priority bandwidth and bypass SIP server(s) to not get billed.

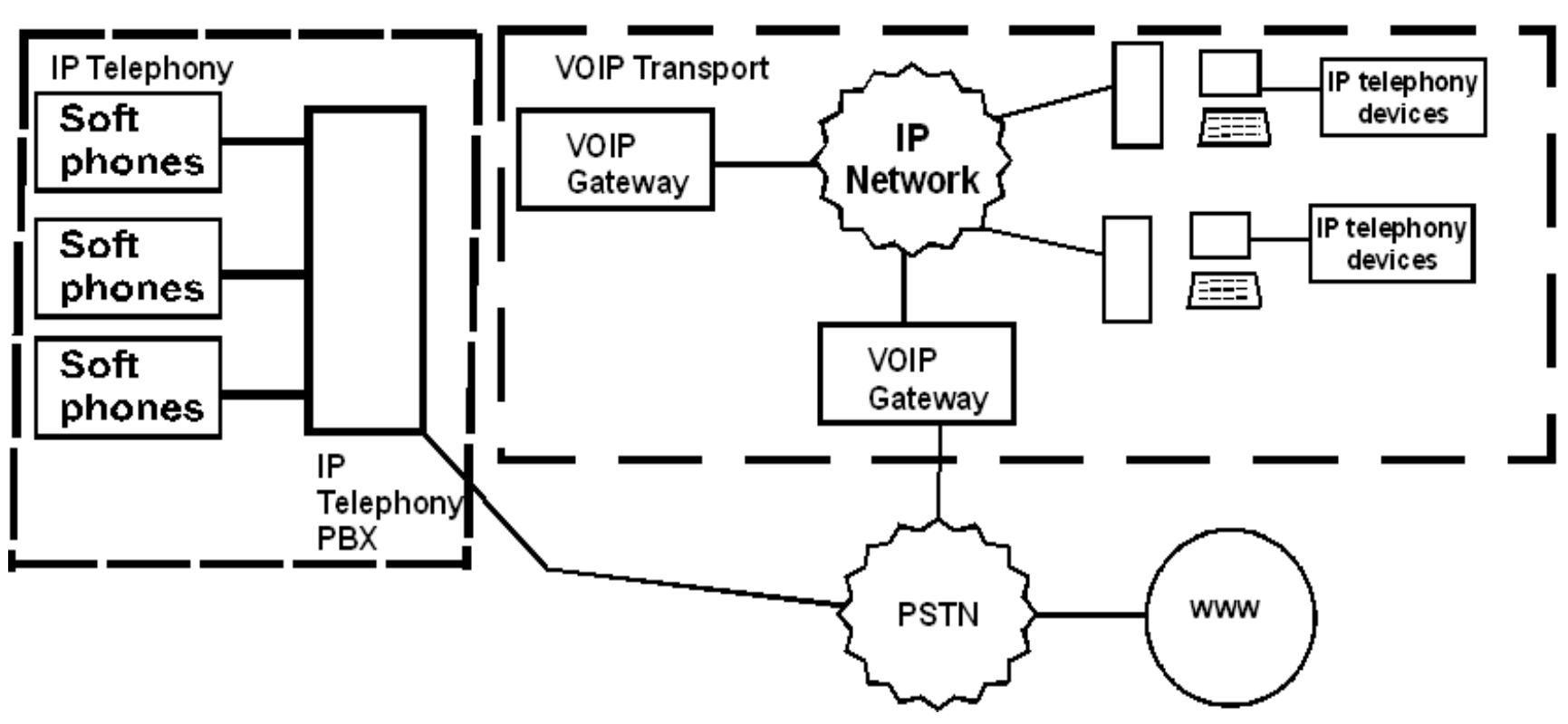

Fig 2. Pragmatic schema of the Hybrid IP/PSTN/PBX Implementation at FUPRE

\subsection{Jitters}

Jitter is difference between the expected time and actual arrive time of packet. Real time voice communications are sensitive to delays and variations in packet arrival times. Codecs require a steady, dependable stream of packets to provide reasonable playback quality. Packets arriving late too early, too late or out of sequence result in jerky and/or jumbled playback. This phenomenon is termed jitters. Since there is no network a perfectly steady stream of packets in real-world apps, soft-phones use buffers to smooth out the problem.

With a constant packet transmission rate of $20 \mathrm{~ms}$ (as in design), new packets is expected to arrive at destination every $20 \mathrm{~ms}$. However, jitters are caused by queuing variations of ongoing changes in traffic loads as well as when one or more packets takes a different equal cost link not physically (electrically) the same length as link used in other voice packets.

To curb this, we employed media with play-out buffer to buffer packet stream so that the reconstructed voice wave is not affected by jitters (or rather minimized). A jitter buffer is simply a First-in, First-Out memory cache that collects the packets as they arrive, forwards them as evenly spaced to the codec and in proper sequence for accurate playback. This enables it to successfully mask mild delays and jitters. Although, sever jitters can also overwhelm the buffer and result in packet loss. Increasing the buffer size can help to a point.

\subsection{Call Management Problem}

The IP call-manager is many times overwhelmed by request or its network connections is impaired, call setup delays can reach the point where users abandon calls before they are able to connect to other party. If a soft-phone is misconfigured or its server connection is impaired, calls remain open in the call queue long after the parties have disconnected.

\subsection{Packet Loss / Bandwidth}

This results from network congestion, router/switch buffer overflow, discarded packets, undesirable file transfer, retransmission ability from protocols etc. Real-time application using RTP protocol for TCP is more tolerant to packet loss than UDP applications. The bandwidth allocated to the net will help a curb it. Network managers can calculate the bandwidth required to support voice traffic, deciding how much to allocate to each service in a converged voice/data network requires careful consideration of an organization's priorities, available bandwidth and its cost. We noted with this implementation, it was noted that packet loss can result from jitter buffers overwhelmed, media failure and poor wireless signal quality such that regardless of its source - the soft-phones, soft agents and gateways attempts to conceal signal degradation by duplicating the packets to fill-in missing data. Packet loss has always been 
characterized as burst phenomena - as the network tends to either sporadically drop single packets called gaps or large number of contiguous packets in a burst. Thus, such factors are considered when making bandwidth calculation as thus:

a. Impact of bandwidth priority

b. Trade-off in compression and voice quality, and

c. Projected peak usage by users

Thus, the selected bandwidth needed support 1,000 full-duplex G.711 encoded voice channels of $20 \mathrm{~ms}$ packet creation and 200 bytes packet size grouped as $(160 \mathrm{~B}$ payload $+40 \mathrm{~B}$ IP header) is thus:

$$
\begin{aligned}
\text { Samples per Secs } & =1000 \mathrm{~ms} / \text { packet creation rate } \\
& =1000 / 20=50 \mathrm{bps}
\end{aligned}
$$

$$
\begin{aligned}
\text { Bps } & =\text { samples } / \text { secs } * \text { packet size } * \text { no calls } * 8 b p s \\
& =50 * 200 * 1000 * 8 \text { bits } / \text { secs }=80 \mathrm{Mbps}
\end{aligned}
$$

Thus, the raw measure of the IP traffic but does not take into account the overhead used by the transport media (links between the routers) and the data link protocol. To determine the link speed needed to support this number of calls, network managers must add this raw IP value to that of the overhead. As implemented, bandwidth requirements vary depending on the rate at which the calls are generated and the signaling protocol used. If a large number of calls are initiated in a relatively short time, the peak bandwidth needs for the signaling can be high. Note the maximum amount of bandwidth required by IP signal protocol is roughly $3 \%$ of all bearer traffic. Thus, 1,000 calls initiated in one second is approximately $2.4 \mathrm{Mbps}$ (i.e $3 \%$ of $80 \mathrm{Mbps}$ ).

\subsection{Network Interconnection}

PSTN is not a single network but a collection of networks operated by various service providers. At each boundary, a network interface is required for interconnection. Interconnection agreements are put in place to cover interconnection points, signaling, timing, billing and tariffs, bearer transport, regulatory requirement etc. In addition, these require approval from the relevant regulators. Scalability constraints and established business model implies that in the nearest future, IP network will become expansive as PSTN (collection of networks and network interconnections will be drawn up).

\subsection{Delays/Latency}

Latency is time taken to reach its destination by the packet through the network. Large delay may not degrade sound quality in phone calls, they can disrupt the rhythm of such conversation - making it difficult to interact. Several factors are known to contribute to such delay in such a multiservice network to include: (a) packet creation, (b) propagation, (c) Serialization, (d) queuing, and (e) packet forwarding.

In designing the multiservice network, total delay a packet exhibits is sum of all latency contributors. An accepted end-to-end latency should be less than $150 \mathrm{~ms}$ for toll quantity calls. To mitigate latency, as implemented at
FUPRE, we have that:

1. Packet Creation Delay is time destination takes to create packets used in the voice services, and exists at both source and destination units of a voice connection. At the source, this delay varies based on amount of time it takes to fill the packet with data. Thus, voice packets tend to be smaller to help minimize amount of delay in the creation process. At destination, media gateway must remove and re-process the packet. Thus, chosen protocol must enforce that all criteria equal, all/any gateway cannot exceed 30ms.

2. Serialization Delay is time taken to serialize digital data onto physical link that interconnects the equipments and it is inversely proportional to the link speed. Thus, the faster the media, the lesser the time it takes to serialize digital data onto physical link and the lower the delay - all of which is dependent on technology used and its access method. Thus, we adopted TCP cable, to keep number of links small and use a high bandwidth interface, to reduce overall delay.

3. Propagation is time a signal takes to travel the conductor's length. Electrical or photonic signal speed via a conductor is always slower than the speed of light - due to propagation delay in net. Computing the delay on $3000 \mathrm{~km}$ (2-way) is:

$P D=\frac{\text { Circuit } k m}{(29300 * 0.6)}=\frac{(3000 * 2)}{(299300 * 0.6)}=33.4$

4. Queuing is packet buffering time as it awaits transfer. It varies on traffic load. Managers can configure length of time packet waits in a network - as this delay is dependent on the amount of traffic network element trying to pass via a given link - and thus, it increases with load. It is curbed with increased bandwidth and resources for voice traffic, as queues not serviced fast enough eventually grows into greater delay.

5. Packet Forward is time it takes router, switch, firewall or other network device to buffer a packet and make a forwarding or drop decision - via accessing which interface to forward the packet to, and whether to drop/forward packet against an Access Control List or security policy. This delay varies and is based on function and architecture of the networked device. So, if a packet is re-buffered as part of processing, the greater the delay incurred.

\subsection{Cost and Reliability}

Certain tradeoffs were considered as adequately explained to management that the cost of this solution cannot equal the cost of implementation of a full-interact access. Thus, the cost of re-access fee and the cost of voice calls services (as used by all staff/students within the institution) will seem expensive. After due consideration, FUPRE's management 
opted for the IP solution with its many benefits to cut down cost of implementation.

PSTN is reliable and handles millions of calls simultaneously - achieved via redundant and load sharing equipment and networks via call agents, access gateways media servers. In addition to fault tolerant feat, equipments, quality - other feats are: (i) no single point of failure, (ii) Hot-swap capability (iii) redundant hardware, (iv) redundant connection and (v) software and/or firmware that are upgraded without loss of service.

\subsection{Quality of Service}

A key requirement to the widespread deployment of VOIP is in its ability to offer toll free quality service equivalence of the existing PSTN. With many users (staff/students) connected, the perceived voice quality became very sensitive in three (3) key performance areas as the network started experiencing: data transfer delays, jitters and packet loss. However, IP by its nature provides a best effort service and does not however, provide guarantee about which key criteria. To $c u b$ this, we implemented ATM (Asynchromous Transfer Mode) -as a means to guarantee prioritization of voice media streams over best-effort data, and to ensure that the VoIP service is not compromised by unforeseen traffic patterns.

\subsection{Bandwidth Utilization}

Using an incremental, implementation approach as earlier noted, gave the researcher a way to improve the quality of service caused by jitters, delays and packet loss - as the researcher (via the ICT unit) decided to reduce system overhead cost by implementing the compression of RTP-UDP (Real-Time Protocol/Universal datagram protocol) and IP headers. A typical sample voice is less than 100 bytes but its combined header equals about 40 bytes-so that for lower bandwidth WAN links such as DSL and cable, the header overhead is significant and reduces the number of voice channels or data bandwidth available. Since a major merit of VOIP is the ability to use lower bit coders so that bandwidth is sowed-this compression mechanism is put in place with a point-to-point link (the goal is to maintain the state for each compressed RTP flow).

\subsection{OSS Support}

PSTN has very extensive Operations Support System providing feats such as: (i) flow through provisioning (ii) fault isolation (iii) loop testing (iv) alarms, (v) performance monitoring and (vi) policy definition and enforcement. Thus, with these in mind - the IP network must be designed to offer the same level of OSS support with PSTN integration via protocols and gateway accesses.

\subsection{Signaling Protocol}

Numerous protocols have been deployed applicable to VOIP. These includes: (a) for device control, we have H248,
MGCP, NCS amongst others, (b) for access service signaling protocol, examples are SIP, H-323 etc, and lastly (c) for network service, examples include SIP, SIP-T, BICC, CMSS etc. The choice of which protocol to use is very much dependent both on the service set being offered alongside the equipment types available to which services. Thus, we implemented the H.248 (Megaco) for device control, H.323 for access service signaling and SIP-T for network service. These were carefully chosen - so that in the event that in the nearest future with upgrades, users include SIP phones, the SIP-T protocol becomes handy.

\section{IP-network Supports}

Managing an IP-based network implies measurement or metrics of some parameters to ensure efficient network running. Thus, the network is and must be subjected to user assessment of quality and monitoring via IP-monitoring tools and analyzers that tends to compute how much various impairments factors like codec compression, jitters, delays and packet loss, will affect the typical user's perception of call and/or service quality. Such support to curb such issues of section 5 is Network Monitoring Tools, that accesses IP quality management from an ICT administrator's view rather than from a telecom engineer - as it aims to track, store and analyze long-term trends, network performance, and maintains a database of Call Detail Records from which the administrator can generate reports for management or service providers. The tools should also be capable to automatically notify when some selected parameter or statistics are developing a problem. An example as used is Network Instrument “Observer". Major components or statistics monitored by the observer includes [11]:

1. Tracks network performance and alerts if MOS (mean option score) or R-factor score falls to 3.5, jitters and delay crosses MPLS mesh of $20 \mathrm{~ms}$ and $80 \mathrm{~ms}$ respectively.

2. Troubleshoots Connection Problems

3. Traffic and call summary

4. Evaluates Jitters

5. Reconstructs and reviews Calls

6. Compares VoIP to network performance

7. Monitors VoWLAN

8. Manages Voice, Video, Audio and Data Quality

9. Monitors Quality of Service (QoS)

10. Measures Bursts and Gaps

11. Tracks and Decodes VoIP and Video

\section{Discussion of Results}

There are lots of benefits accrued to this hybrid implementation as proposed, and the nature of this work compelled the researchers to join the Multiservice Switching Forum (MSF), which is committed to aggressive technical solutions for a full PSTN replacement network with next generation IP infrastructure as well as QoS cum security in a way that scales to the many billions of busy hour calls that a 
typical PSTN must handle.

MSF's approach to problem solving: (i) proposes a coherent and pragmatic network vision (ii) identifies existing protocols that delivers its vision, enhance them for the purpose while eradicating barriers to easy interoperability, and (iii) develop program of easily interoperability testing, with carrier grade equipment supported by detailed to relevant test plans.

Frequent reviews are released (though a major reason for implementing our incremental approach design/model - so as to accommodate the problems as they surfaced in bits). Other issues currently undertaken by MSF are:

1. Scaleable IP-QoS (voice/multimedia) over IP defines a solution to provide scalable QoS to the many billions of BHCA that PSTN supports in order to create an interaction between SIP services and QoS mechanism that will address the finality required in IP edge router.

2. Security - end-to-end IP implies vulnerability to a variety of attacks as mentioned. This can be addressed using cryptographic schemes.

3. Service/Feat Interactions - MSF service layer will aim to identify and prove new innovative network services and verifying that the services can be supported over such scalable next generation VOIP,

4. Call-routing mechanisms are to be considered a great power when implementing pure IP, mixed IP and/or legacy PSTN network,

5. Management related issues will hinge on a design that will motivate the overall architecture and act as a start point for protocol profiling and interoperability testing.

\section{Summary / Conclusion}

IP is ubiquitous and cost-effective. Thus, my moving towards an IP-based network, a carrier can:

1. Managing Call Quality:

2. Deploy new voice/data services, removing need to manage separate voice and data networks.

3. Reap the benefits of a standard, highly flexible network, giving competitive market to equipment vendors, and encompassing a wide range of equipment for different market niches.

4. Utilize cheaper IP-based backbone equipments to carry voice data.

A number of IP solutions exists with limitations that are dependent on solution type, bandwidth etc. These can be attributed to implementation around early versions of standards that provide restricted interoperability with and between other vendors.

\section{Recommendations}

[12-13] note these recommendations:

1. To improve service quality, network administrators and managers must: (a) Understand and measure call quality component, (b) conduct site surveys, (c) deploy analysis tools strategically for maximum visibility, (d) implement quality of service prioritization, (e) implement VLAN to isolate/monitor VoIP issues, (f) monitor rollouts to ensure positive user experience, $(\mathrm{g})$ compare jitters to overall network bandwidth utilization to understand network response time, (h) set up analyzer to automatically and proactively monitor VoIP activities, (i) automate problem resolution and (j) set up baseline network traffic.

2. MSF (multiservice switching forum) is committed to providing next generation network that provides both full multi-vendor interoperability as well as the support for a full featured, secure PSTN. Thus, institutions seeking such solutions are encouraged to register with MSF so that their personnel can take advantage of such forum in proffering solutions to issues in the implementation of their solution type.

\section{References}

[1] Abraham, M., Jajodia, S and Podell, H., "Data security", IEEE Transaction on Comp., 1995, 13(2), CA: Los Alamitos.

[2] Brennen, R and Dipak, G., "Secure IP telephony via multi-layered protection", 2009, Technical University of Denmark report, Centre for ICT, Denmark: Lyngby.

[3] Drew, P and Gallon, C., "Next generation VoIP network architecture: MSF whitepaper report", 2003, www.msforum.org, accessed April 2013.

[4] Eung-Ha, K., Cho, K.S and Ryu, W., "Amendment to MSF whitepaper on personalized converged services: network-to-network", www.msforum.org/techninfo/reports.shtml, last accessed March 2013.

[5] Garg, V., Smolik, K and Wilkes, J., "CDMA application in wireless communications", 1997, Prentice Hall publications, New Jersey: Upper Saddle River.

[6] Hafner, K and Lyon, M., "Where wizards stay up late", 1996, Simon and Schuster, New York.

[7] Helgert, H., "Integrated services digital net: Architecture, protocols and standards", 1991, Readings: MA: Addison-Wesley.

[8] International Telecommunications Union Supplement Handbook., "Rural telecommunications", 2010, pp12-48, Geneva.

[9] Martins, J.A.H., "Telecommunications and the computer", 1990, Prentice Hall publications, New Jersey: Upper Saddle river

[10] Matthews, V., Shakunle, J and Adetiba, E., "Hybrid cellular mobile network for rural telecommunications in Nigeria", 2007, Int. J. Research in Physical Sci., 
ISSN: 1597-0823, 4(1), pp 34-42.

[11] Network Instrument., "A White paper guide to troubleshooting IP-based networks, 2007, [online]: www.networkinstruments.com.

[12] Ojugo, A.A, "Introducing VoIP in Nigeria", 2010, J. Res. Phy. Sci., ISSN-1597-8028, 6(1), pp 43-51.

[13] Ojugo, A.A., Yoro, E.R., Eboka, A.O., Yerokun, M.O and Iyawa, I.J.B., "Implementation issues of VoIP for rural telephony in Nigeria", Journal of Emerging Trends in Comp. and Information System, ISSN: 2079-8407, 2012, 4(2), pp 172-179, [online]:www.cisjournals.org

[14] Osuagwu, O.E., Anyanwu, E and Amaeshi, L., "Deployment of computer-assisted radar technology: a case study for improved military security and surveillance in Nigeria", 2005, J. Computer Sci. App., $9(1)$, pp.71-81.

[15] Rosa, J., "Rural telecommunications via satellite", J. of Telecommunications, 2005, 3(2), pp $75-81$.

[16] Schwartz, R., "Wireless communications in developing nations: cellular and satellite net systems", 1997,
Artech house. MA: Boston.

[17] Stallings, W and Van Slyke, R., "Business data communications", 6th Ed., 2010, Prentice Hall Int. Ed., ISBN - 1-13-761230-3, pp316-340.

[18] Williams, M., Ewan, S and Reza, T., "Convergence, IP telephony and regulations: issues and opportunities for network development in India”, 2005, Technical Univeristy of Denmark Center for ICT, Denmark: Lyngby.

[19] Conte, R., "Rural telephony: new appraoch via mobile satellite communication", Proceedings of Pacific telemmunication conf., 1994, Hawai: Honolulu.

[20] Salamsi, A and Gilhousen, K.S., "On system design aspects of CDMA applied to digital cellular and personal communication network", 1991, IEEE Proc. Vehicular Tech. Conference, pp 57-63.

[21] Yoro, E.R., "Next Generation VoIP deployment: a case study of Delta State Polytechnic Ogwashi-Uku “, 2012, an unpublished Masters thesis, Department of Computer Science, Benson Idahosa University: Benin City, Nigeria. 